\title{
Nanosecond difference-frequency-generation in orientation-patterned gallium phosphide
}

\author{
Junxiong Wei, ${ }^{1}$ S. Chaitanya Kumar, ${ }^{1,2,}{ }^{*}$ Hanyu Ye, ${ }^{1}$ Kavita Devi, ${ }^{1}$ Peter G. \\ SChunemanN, ${ }^{3}$ M. EBRAHIM-ZADEH ${ }^{1,2,4}$ \\ ${ }^{1}$ ICFO-Institut de Ciencies Fotoniques, The Barcelona Institute of Science and Technology, 08860 Castelldefels (Barcelona), Spain \\ ${ }^{2}$ Radiantis, Polígon Camí Ral, 08850 Gavà, Barcelona, Spain \\ ${ }^{3}$ BAE Systems, Incorporated, MER15-1813, P.O. Box 868, Nashua, New Hampshire 03061-0868, USA \\ 4 Institucio Catalana de Recerca i Estudis Avancats (ICREA), Passeig Lluis Companys 23, Barcelona 08010, Spain \\ *Corresponding author: chaitanya.suddapalli@radiantis.com
}

Received XX Month XXXX; revised XX Month, XXXX; accepted XX Month XXXX; posted XX Month XXXX (Doc. ID XXXXX); published XX Month XXXX

We report a tunable, single-pass, pulsed nanosecond difference-frequency-generation (DFG) source based on the new semiconductor nonlinear material, orientationpatterned gallium phosphide (OP-GaP). The DFG source is realized by mixing the output signal of a nanosecond OPO tunable over 1723-1827 $\mathrm{nm}$ with the input pump pulses of the same OPO at $1064 \mathrm{~nm}$ in an OP-GaP crystal, resulting in tunable generation over $233 \mathrm{~nm}$ in the midinfrared from 2548 to $2781 \mathrm{~nm}$. Using a 40-mm-long crystal, we have produced $\sim 14 \mathrm{~mW}$ of average DFG output power at $2719 \mathrm{~nm}$ for a pump power of $5 \mathrm{~W}$ and signal power of $1 \mathrm{~W}$ at $80 \mathrm{kHz}$ repetition rate. To the best of our knowledge, this is the first single-pass nanosecond DFG source based on OP-GaP. The DFG output beam has a TEMoo spatial mode profile and exhibits passive power stability better than $1.7 \%$ rms over 1.4 hours at $2774 \mathrm{~nm}$, compared to $1.6 \%$ and $0.1 \%$ rms for the signal and pump, respectively. The $\mathrm{OP}-\mathrm{GaP}$ crystal is recorded to have a temperature acceptance bandwidth of $17.7^{\circ} \mathrm{C}$.

OCIS codes: (160.4330) Nonlinear optics, materials; (190.4410) Nonlinear optics, parametric processes; (140.6810) Nonlinear optics, devices.

http://dx.doi.org/10.1364/OL.99.099999

Mid-infrared (Mid-IR) pulsed sources in the 2-3 $\mu \mathrm{m}$ wavelength range are highly desirable for industrial, medical and securityrelated applications [1]. Access to this spectral region can be achieved by using conventional bulk/fiber lasers as well as optically pumped semiconductor lasers [2-4]. However, stable, high output power together with spectral agility can be best achieved by nonlinear frequency conversion techniques, such as difference-frequency-generation (DFG) and optical parametric oscillators (OPOs). While OPOs can provide tunability together with high efficiency, DFG sources can be realized in simple singlepass architecture. The ability to tune one of the input beams in the
DFG process results in tunable output at longer wavelengths. Such DFG sources based on near-IR nonlinear materials such as $\beta$ $\mathrm{BaB}_{2} \mathrm{O}_{4}$ (BBO) [5], $\mathrm{KTiOPO}_{4}$ (KTP) [6] and MgO-doped periodically poled $\mathrm{LiNbO}_{3}$ (MgO:PPLN) [7], and mid-IR nonlinear materials such as $\mathrm{ZnGeP}_{2}$ (ZGP) [8] and orientation-patterned GaAs (OPGaAs) [9] have been previously demonstrated. Although ZGP and OP-GaAs can provide spectral coverage deep into the mid-IR, they require pumping beyond $2 \mu \mathrm{m}$ due to two-photon absorption at short-wavelength transparency cut-off [10]. On the other hand, BBO, PPKTP and MgO:PPLN can be pumped using the readily available near-IR laser sources in the 1-2 $\mu \mathrm{m}$ range, but their longwavelength transparency cut-off limits efficient DFG beyond $\sim 4$ $\mu \mathrm{m}$. As such, it is important to investigate alternative nonlinear materials, which can be pumped in the near-IR to generate deep mid-IR radiation. One such material is the recently developed birefringent nonlinear crystal, $\mathrm{CdSiP}_{2}$ (CSP), which enables DFG at wavelengths as long as $6 \mu \mathrm{m}$ when pumped in the near-IR [11]. Orientation-patterned gallium phosphide (OP-GaP) is a new quasiphase-matched (QPM) nonlinear material with transparency across $0.8-12 \mu \mathrm{m}$ and a high nonlinear coefficient of $\sim 70 \mathrm{pm} / \mathrm{V}$ [12]. It also possesses a high thermal conductivity of $110 \mathrm{~W} / \mathrm{m}-\mathrm{K}$, leading to a high damage threshold of $0.8 \mathrm{~J} / \mathrm{cm}^{2}$. Its wide transparency range, with short-wavelength cut-off extending below $1 \mu \mathrm{m}$ enables the deployment of the well-established Ndbased solid-state or Yb-based fiber pump laser technology for efficient frequency conversion into the mid-IR. Moreover, the QPM condition can be engineered by orientation-patterning the required grating period, enabling wavelength conversion over the full transparency range of the material.

Earlier reports on nonlinear frequency conversion in OP-GaP include a nanosecond doubly-resonant OPO (DRO) pumped at $1064 \mathrm{~nm}$, generating $4 \mathrm{~mW}$ of idler at $4624 \mathrm{~nm}$ and $15 \mathrm{~mW}$ of signal at $1324 \mathrm{~nm}$ at $10 \mathrm{kHz}$ [13], and a nanosecond DRO pumped at $2090 \mathrm{~nm}$ operating at a fixed idler wavelength of $5100 \mathrm{~nm}$ and a signal wavelength of $3540 \mathrm{~nm}$, providing a total signal plus idler output power of $350 \mathrm{~mW}$ at $20 \mathrm{kHz}$ [14]. In the continuous-wave 
(cw) regime, single-pass DFG based on a 16.5-mm-long OP-GaP crystal was demonstrated, generating up to $150 \mathrm{~mW}$ of DFG power at $3400 \mathrm{~nm}$ for an input pump power of $47 \mathrm{~W}$ at $1064 \mathrm{~nm}$, together with $24 \mathrm{~W}$ of input signal power at $1550 \mathrm{~nm}$ [15]. Recently, a cw DFG power of $65 \mu \mathrm{W}$ was generated at $5850 \mathrm{~nm}$ in a 24.6-mm-long OP-GaP crystal for pump power of $10 \mathrm{~W}$ at 1064 $\mathrm{nm}$ and an input signal power of $40 \mathrm{~mW}$ at $1301 \mathrm{~nm}$ [16].

In this Letter, we report a tunable DFG source based on OP-GaP operating across $2548-2781 \mathrm{~nm}$. While a commercial Q-switched nanosecond laser at $1064 \mathrm{~nm}$ serves as the pump, the input signal beam for the DFG is provided by a pulsed MgO:PPLN OPO pumped by the same laser. The DFG source can provide up to $\sim 14 \mathrm{~mW}$ of average output power at $2719 \mathrm{~nm}$ at $80 \mathrm{kHz}$ repetition rate, with $>6 \mathrm{~mW}$ across the full tuning range, in TEMoo spatial profile, with a passive power stability better than $1.7 \%$ rms over 1.4 hours.

The schematic of the experimental setup for single-pass DFG in OP-GaP is shown in Fig. 1 . The primary pump source is a Qswitched Nd:YAG laser (Bright Solutions, Sol), delivering up to 30 $\mathrm{W}$ of average power at $1064.7 \mathrm{~nm}$ in linear polarization, with tunable repetition rate. The output pulse width varies from $8 \mathrm{~ns}$ at $20 \mathrm{kHz}$ to $26 \mathrm{~ns}$ at $100 \mathrm{kHz}$ repetition rate. However, for the experiments presented in this report, we used a repetition rate of $80 \mathrm{kHz}$, with a corresponding pump pulse duration of $23 \mathrm{~ns}$. The instantaneous bandwidth and frequency jitter of this laser, measured using an optical spectrum analyzer with a resolution $<0.5 \mathrm{~nm}$, is $\sim 0.2 \mathrm{~nm}$ and $\sim 1 \mathrm{~nm}$ over 30 seconds, respectively. A major portion of the output power from the laser is used to pump a pulsed OPO which provides the input signal beam for DFG in OP$\mathrm{GaP}$, while the remaining output is

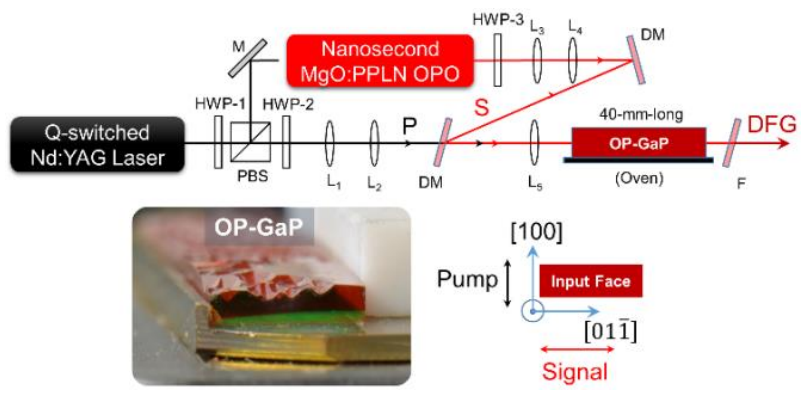

Fig. 1. Schematic of the experimental setup. HWP: Half-wave-plate, PBS: Polarizing beam-splitter, DM: dichroic mirror, M: Mirrors, L: Lens, F: Germanium filter, S: signal beam, P: pump beam.

used as the pump for DFG. The nonlinear gain medium for the pulsed OPO is a 38-mm-long, 1-mm-thick, MgO:PPLN crystal, with five gratings ranging in period from $\Lambda_{\mathrm{OPO}}=29.5$ to $31.5 \mu \mathrm{m}$ in steps of $0.5 \mu \mathrm{m}$. However, in the present experiments we used a single grating period of $\Lambda_{\mathrm{OP} 0}=31.5 \mu \mathrm{m}$. The end faces of the crystal are antireflection (AR)-coated $(R<4 \%)$ for the pump and idler, with high transmission $(R<1 \%)$ for the signal over $1300-1900 \mathrm{~nm}$. The crystal is housed in an oven, which can be adjusted from room temperature to $200{ }^{\circ} \mathrm{C}$, with a stability of $\pm 0.1{ }^{\circ} \mathrm{C}$. The OPO is configured as a singly resonant oscillator for the signal in a threemirror cavity. In order to partially extract the signal from the cavity, we used $\sim 22 \%$ output coupler, while the idler was extracted in single pass. The OPO can be temperature-tuned over $1664-1928 \mathrm{~nm}$ in the signal and 2374-2950 $\mathrm{nm}$ in the idler for a fixed grating period of $\Lambda_{\mathrm{OPO}}=31.5 \mu \mathrm{m}$.
The power scaling performance of the MgO:PPLN nanosecond OPO is shown in Fig. 2. The variation of the signal power, extracted through the $22 \%$ output coupler at the wavelength of $1730 \mathrm{~nm}$, together with the simultaneously measured idler power at 2763 $\mathrm{nm}$, is depicted in Fig. 2. For a maximum average pump power of $10 \mathrm{~W}$ at $80 \mathrm{kHz}$, we were able to extract up to $2.16 \mathrm{~W}$ of signal together with $0.92 \mathrm{~W}$ of idler power, corresponding to a total power of $3.08 \mathrm{~W}$ from the OPO, at an overall extraction efficiency of $\sim 31 \%$. The signal and idler slope efficiencies are estimated to be

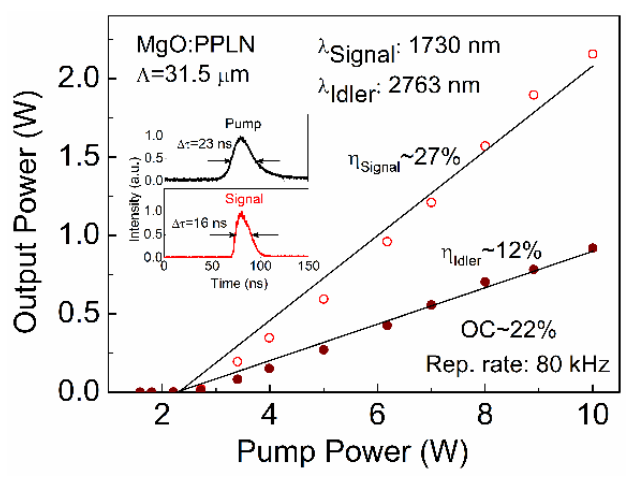

Fig. 2. Power scaling of the MgO:PPLN pulsed OPO at a signal wavelength of $1730 \mathrm{~nm}$ using $22 \%$ output coupler. Inset: Pulse shapes of the incident pump, and signal pulses.

$\sim 27 \%$ and $\sim 12 \%$, respectively. The average pump power threshold of the OPO was recorded to be $1.6 \mathrm{~W}$. Also shown in the inset of Fig. 2 is the pump and signal pulse durations, measured using an InGaAs photodetector. The full-width-half-maximum (FWHM) signal pulse duration is $16 \mathrm{~ns}$, which is shorter than the pump pulse duration of $23 \mathrm{~ns}$, as expected. Hence, we have $>2 \mathrm{~W}$ of usable average signal power for DFG at the output of the OPO.

The input pump and signal beams for DFG are collimated and combined using a dichroic mirror, DM, which is AR-coated for high reflection $(R>99 \%)$ for the signal over $1300-2000 \mathrm{~nm}$ and high transmission (T>90\%) for the pump at $1064 \mathrm{~nm}$. The pump and signal beams are then focused using a single lens, L5, of focal length $f=250 \mathrm{~mm}$, to achieve beam waist radii of $w_{\mathrm{op}} \sim 65 \mu \mathrm{m}$ and $w_{\mathrm{os}} \sim 60$ $\mu \mathrm{m}$, corresponding to a focusing parameter of $\xi_{\mathrm{P}} \sim 0.5$ and $\xi_{\mathrm{S}} \sim 1$, for the pump and signal, respectively, at the center of the OP-GaP crystal. This results in an estimated DFG beam waist radius of $w_{\mathrm{DFG}} \sim 44 \mu \mathrm{m}$, with a focusing parameter of $\xi_{\mathrm{DFG}} \sim 3$. The OP-GaP crystal for DFG is a 40-mm-long, 6-mm-wide, 1.7-mm-thick, with a grating period of $\Lambda_{\mathrm{DFG}}=15.5 \mu \mathrm{m}$. The end faces of the crystal are ARcoated for high transmission $(R<1 \%)$ at $1064 \mathrm{~nm}$ and 1500-1900 $\mathrm{nm}$, with $>80 \%$ transmission over $2500-2800 \mathrm{~nm}$. The OP-GaP crystal, shown in the inset of Fig. 1, is similarly housed in an oven, which can be adjusted from room temperature to $200^{\circ} \mathrm{C}$. The DFG output is separated from the near-IR pump and signal beams using a coated Ge filter with a transmission of $\sim 90 \%$ for DFG. The polarization dependence for three-wave mixing in OP-GaP crystal has been described in [15], where the general expression for the effective second-order nonlinear coefficient, $d_{\mathrm{eff}}$, has been derived. Here, the pump and signal polarizations are set along [100] (vertical) and [011] (horizontal) directions, respectively. Further, two half-wave plates, HWP-2 and HWP-3, are used to provide independent control of the pump and signal polarizations for phase-matching optimization to achieve maximum DFG power. 
The dichroic mirrors are used to optimize spatial overlap of the pump and signal beams. The OP-GaP crystal is mounted on a three-axis stage to optimize the position of the input beams in the crystal for maximum DFG power. Initially, we observed parasitic generation of red light as a result of non-phase-matched sumfrequency-generation (SFG) between the pump and the input signal, which was also useful in optimizing the DFG power as well as determining of signal and DFG output wavelengths.

In order to characterize the DFG source based on OP-GaP crystal, we performed spectral tuning by varying the OPO signal wavelength. We varied the temperature of the Mg0:PPLN
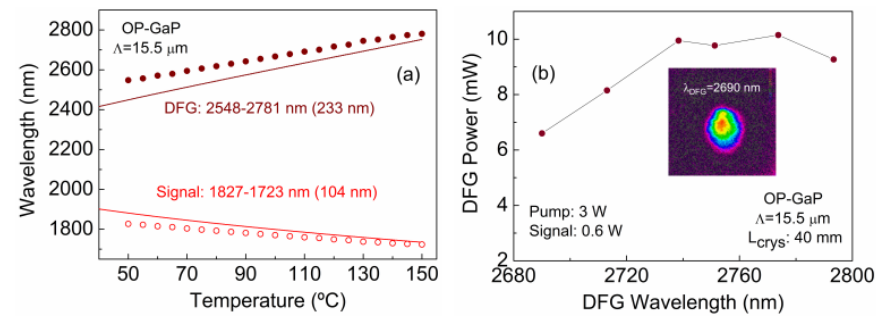

Fig. 3.(a)DFG wavelength tuning range as a function of crystal temperature for a grating period of $\Lambda_{\mathrm{DFG}}=15.5 \mu \mathrm{m}$ in OP-GaP. Solid circles represent experimental data, while the solid lines are theoretical calculations. (b) DFG output power across the tuning range. Inset: Spatial beam profile of the generated DFG beam.

crystal, and simultaneously adjusted the phase-matching temperature of the OP-GaP crystal at a fixed pump power of $3 \mathrm{~W}$ and a signal power of $0.6 \mathrm{~W}$. The signal and DFG wavelengths were inferred from the non-phase-matched parasitic SFG of the signal and pump, measured using a visible spectrometer with a resolution of $0.5 \mathrm{~nm}$ (Ocean Optics, HR4000). Figure 3(a) shows the DFG wavelength tuning results. By varying the temperature of the MgO:PPLN crystal from $105^{\circ} \mathrm{C}$ to $170^{\circ} \mathrm{C}$, and the temperature of OP-GaP from $150{ }^{\circ} \mathrm{C}$ to $50{ }^{\circ} \mathrm{C}$, we were able to achieve tunable DFG across $2548-2781 \mathrm{~nm}$ in the mid-IR. The DFG wavelength varies at a rate of $2.4 \mathrm{~nm} /{ }^{\circ} \mathrm{C}$. Here, the circles represent the experimental data, while the solid curves correspond to calculated temperature tuning range in OP-GaP using the relevant Sellemier equations [13]. As evident from the plot, we were able to obtain DFG spectral coverage over $233 \mathrm{~nm}$ in the mid-IR by tuning the OPO signal wavelength over $104 \mathrm{~nm}$. The small discrepancy between the theoretical calculation and experimental data is attributed to the difference in the actual temperature of the OP$\mathrm{GaP}$ crystal and the set oven temperature. We also recorded the single-pass DFG power obtained across the tuning range of 2690$2793 \mathrm{~nm}$, with the results shown in Fig. 3(b). For each signal wavelength, we optimized the temperature of the OP-GaP crystal to obtain maximum DFG power. The source could provide up to $10.2 \mathrm{~mW}$ of mid-IR DFG power at $2774 \mathrm{~nm}$ for $3 \mathrm{~W}$ of pump and $0.6 \mathrm{~W}$ of signal power, with $>6 \mathrm{~mW}$ available over the entire tuning range. The inset of Fig. 3(b) shows the far-field energy distribution of the DFG output beam at $2690 \mathrm{~nm}$, at maximum power, recorded using a pyroelectric camera. The result indicates TEMo0 profile with a single peak Gaussian distribution. Similar beam profiles were observed across the entire DFG tuning range.

In order to understand the tolerance of the generated DFG power to the temperature, we investigated the temperature acceptance bandwidth for DFG in the OP-GaP crystal. We performed the measurements at a fixed pump power of $3 \mathrm{~W}$ and a signal power of $0.6 \mathrm{~W}$ to enable accurate determination of the DFG power. The normalized DFG power as a function of the phasematching temperature, together with the $\sin c^{2}$ fit, is shown in Fig. 4. The measurement resulted in a FWHM temperature acceptance bandwidth of $\Delta \mathrm{T} \sim 17.7 \stackrel{\circ}{\circ} \mathrm{C}$. This is much wider than the theoretically estimated bandwidth of $\Delta \mathrm{T}=1{ }^{\circ} \mathrm{C}$ for a 40 -mm-long OP-GaP crystal under plane-wave approximation, using the relevant temperature-dependent Sellmeier equations [13], as shown in the inset of Fig. 4. It is to be noted that the theoretically calculated phase-matching temperature is $\sim 124.4{ }^{\circ} \mathrm{C}$, which is substantially different from the experimental value. The difference in the phase-matching temperature and the broader temperature acceptance bandwidth could be attributed mainly to the wide spectral bandwidth of pump and signal beams, as well as nonoptimal focusing and thermal effects.

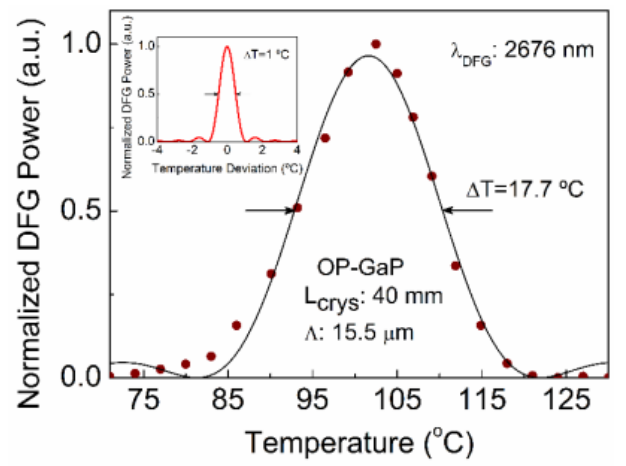

Fig. 4. Experimentally measured, and (Inset) theoretically calculated temperature acceptance bandwidth for a 40-mm-long OP-GaP crystal.

The power scaling results for DFG in OP-GaP are presented in Fig. 6. For a fixed signal power of $1 \mathrm{~W}$ at $1748 \mathrm{~nm}$, and increasing the pump power at the input to the OP-GaP crystal up to $5 \mathrm{~W}$, we were able to generate a maximum of $13.8 \mathrm{~mW}$ at $2719 \mathrm{~nm}$. The DFG power increases linearly at a slope efficiency of $0.3 \%$, as shown in Fig. 5(a). Further, we also measured the transmission of the OP-GaP crystal for the input beams at the same position where we generated the maximum DFG power. The transmission for the pump and signal beams, while producing the DFG, were recorded to be $28 \%$ and $18 \%$, respectively. Considering the losses in the OPGaP crystal, the generated maximum DFG power represents a pump to DFG conversion efficiency of $\sim 1 \%$, corresponding to a photon conversion efficiency of $\sim 2.5 \%$. A similar measurement for a fixed pump power of $5 \mathrm{~W}$, and varying the signal power, is shown in Fig. 5(b), where the DFG power is seen to increase linearly with a slope efficiency of $1.2 \%$, providing a maximum DFG power of $13.8 \mathrm{~mW}$ at $1 \mathrm{~W}$ of signal power. It is to be noted that the DFG powers presented here are not corrected for the losses in the crystal and the separating filter. While performing the power scaling measurements, the temperature of OP-GaP crystal was adjusted to achieve the maximum DFG power. As the pump power was increased from the $0.6 \mathrm{~W}$ to $5 \mathrm{~W}$, the phase-matching temperature of the $\mathrm{OP}-\mathrm{GaP}$ had to be reduced from $119{ }^{\circ} \mathrm{C}-150 \stackrel{\circ}{ } \mathrm{C}$, indicating strong thermal effects. This change in temperature is much wider than the measured temperature acceptance bandwidth presented in Fig. 4, and can be attributed to the absorption at the pump and signal wavelengths, resulting in the 
temperature rise in the OP-GaP crystal, thereby necessitating the reduction of the phase-matching temperature.

Further, we estimated the effective nonlinear coefficient, deff, from the power scaling data. Using simple DFG theory, a $d_{e f f} \sim 13$ $\mathrm{pm} / \mathrm{V}$ is estimated, close to recently reported

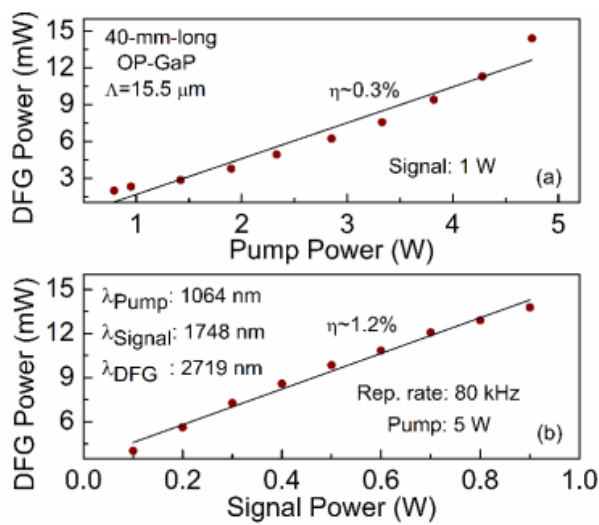

Fig. 5. Dependence of the measured DFG output power at $2719 \mathrm{~nm}$ on (a) incident pump power with fixed OPO signal power, and (b) incident OPO signal power with fixed pump power.

value of $17 \mathrm{pm} / \mathrm{V}$ [16]. However, the contributions of finite pump and signal spectral bandwidths in our experiment, as well as thermal effects due to the absorption of pump, signal and idler in the present crystal, lead to a lower estimate of $d_{\text {eff }}$ for the material.
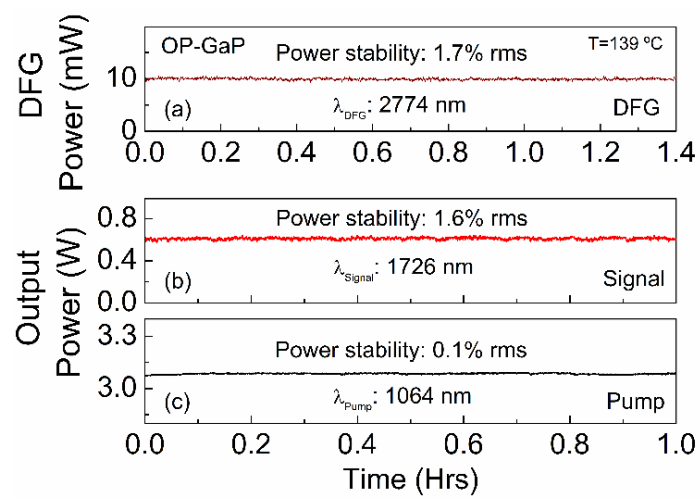

Fig. 6. Long-term power stability of the (a) DFG output beam at maximum pump power of $3 \mathrm{~W}$ over 1.4 hours, simultaneously measured (b) signal and (c) pump power stability.

We also recorded the long-term passive power stability of the DFG source under free-running conditions, with the results shown in Fig. 6(a). The generated mid-IR DFG power measured at a central wavelength of $2774 \mathrm{~nm}$ was recorded to exhibit a passive stability better than $1.7 \%$ rms over 1.4 hours, as compared to the simultaneously measured signal and pump power stability of $1.6 \%$ and $0.1 \%$ rms over 1 hour measured at $1726 \mathrm{~nm}$ and $1064 \mathrm{~nm}$, respectively, as shown in Fig. 6(b,c).

In conclusion, we have demonstrated a tunable nanosecond pulsed single-pass DFG source for the mid-IR based on the new semiconductor nonlinear material, OP-GaP. Using a Q-switched $\mathrm{Nd}$ :YAG pump laser at $1064 \mathrm{~nm}$, the DFG output wavelength is tuned by simultaneously varying the signal wavelength from a pulsed Mg0:PPLN OPO pumped by the same laser and the phasematching temperature of the OP-GaP crystal, providing tunability over $233 \mathrm{~nm}$ in the mid-IR across 2548-2781 nm. The source can provide a maximum DFG power of $\sim 14 \mathrm{~mW}$ at $80 \mathrm{kHz}$ repetition rate for an average pump power of $5 \mathrm{~W}$ and signal power of $1 \mathrm{~W}$. The 40-mm-long OP-GaP crystal has been measured to have a temperature acceptance bandwidth of $17.7^{\circ} \mathrm{C}$. High transmission loss in the OP-GaP crystal at the pump and signal wavelengths has been observed, leading to strong thermal effects due to absorption. Further enhancements in the DFG power are expected with improved quality of the OP-GaP crystal in terms of transmission at shorter wavelengths and duty cycle of the QPM grating period. The DFG output beam has been recorded to exhibit a passive power stability better than $1.7 \%$ rms over 1.4 hours at $2774 \mathrm{~nm}$, as compared to $1.6 \%$ and $0.1 \% \mathrm{rms}$ for the signal and pump respectively, in TEM $\mathrm{O}_{00}$ spatial beam profile.

Acknowledgment. We acknowledge support from Ministerio de Economía y Competitividad (MINECO) (nuOPO, TEC2015-68234R); European Commission (EC) (H2020-MSCA-ITN-2014); Agència de Gestió d'Ajuts Universitaris i de Recerca (AGAUR) (SGR 2014-2016); Severo Ochoa (SEV-2015-0522); Fundación Cellex. Hanyu Ye acknowledges the support of Marie Curie Actions: Innovative Training Network through the Mid-Tech project (H2020-MSCA-ITN-2014).

\section{References}

1. M. Ebrahim-Zadeh and I. T. Sorokina, Mid-infrared Coherent Sources and Applications (Springer, 2008)

2. J. Nicolas, A. N. Baranov, Y. Cuminal, Y. Rouillard, and C. Alibert, Appl. Opt. 37, 7906 (1998)

3. X. Zhu and R. Jain, Opt. Lett. 32, 2381 (2007)

4. A. P. Ongstad, G. C. Dente, M. L. Tilton, R. Kaspi, IEEE J. Sel. Top. Quantum Electron. 19, 1900608 (2013)

5. S. Ashworth, C. laconis, O. Votava, E. Riedle, Opt. Commun. 97, 10914 (1993)

6. G. C. Bhar, A. M. Rudra, A. K. Chaudhary, T. Sasaki, Y. Mori, Appl. Phys. B 63 141 (1996)

7. J. Saikawa, M. Miyazaki, M. Fujii, H. Ishizuki, and T. Taira, Opt. Lett. 33, 1699-1701 (2008)

8. K. Zhong, J. Li, D. Xu, J. Wang, Z. Wang, P. Wang, J. Yao, Opto electron. Lett. 6, 179 (2010)

9. K. Devi, P. G. Schunemann, and M. Ebrahim-Zadeh, Opt. Lett. 39, 6751 (2014)

10. V. Petrov, Prog. Quantum Electron. 42, 1 (2015)

11. S. Chaitanya Kumar, P. G. Schunemann, K. T. Zawilski, and M. EbrahimZadeh, J. Opt. Soc. Am. B 33, D44-D56 (2016)

12. P. G. Schunemann, K. T. Zawilski, L. A. Pomeranz, D. J. Creeden, and P. A. Budni, J. Opt. Soc. Am. B 33, D36 (2016)

13. L. A. Pomeranz, P. G. Schunemann, D. J. Magarrell, J. C. McCarthy, K. T. Zawilski, and D. E. Zelmon, Proc. SPIE 9347, 93470K (2015)

14. Peter G. Schunemann, Leonard A. Pomeranz, and Daniel J. Magarrell, Proc. SPIE 9347 93470J (2015)

15. S. Guha, J. O. Barnes, P. G. Schunemann, Opt. Mater. Express 5, 2911 (2015)

16. G. Insero, C. Clivati, D. D’Ambrosio, P. De Natale, G. Santambrogio, P. G. Schunemann, J. J. Zondy, and S. Borri, Opt. Lett. 41, 5114 (2016) 